\title{
THE VIBRATION ANALYSIS BY MARGENAU-HILL TRANSFORMATION METHOD
}

\begin{abstract}
The paper is devoted to the description and application of Margenau Hill transformation for vibration analysis. This is a transformation belonging to the large group of non-linear time-frequency transformations. The properties predestinated it to a successful use in the area of non-stationary and transitional signal analysis describing various natural processes. A very interesting application area of this transformation is its use for the vibration analysis of signals from railway traffic. The paper contains mathematical analysis of the transformation, case study and gained practical experience and recommendations for practice.
\end{abstract}

Keywords: Vibration, signal analysis, time frequency transformation, railway transport, crossing construction.

\section{Introduction}

The information on any engineering, physical, or other phenomenon is represented in the signal by the changes over time of the current value of the quantity described by the signal. In many applications, direct evaluation of the time-amplitude representation is neither easy nor advantageous. For this reason, at first, the signal is transformed from the time-domain into another one. In some cases, important information can be obtained from the frequency domain. For the transformation from time to frequency area the most used and known method is the Fourier's transformation and their modifications. The Fourier transform, or its modification and some of the parametric methods are well suited techniques to processing stationary signals.

For determination of time localization of frequency components within non-stationary signals it is not possible to use classical proceedings of frequency analyses but it is necessary to apply some other transformation procedures and other calculation methods.

\section{Time frequency analysis}

If the information sources from the time and frequency domains are to be combined, we can use what is called timefrequency transformations. This enables us to determine the frequency as a function of time. So, one of the possible procedures is the application of so called time-frequency transformations.
They can be divided according to calculating methods into two basic classes [1]:

- linear (including especially short-time Fourier's transformation, transformation Wavelet etc.)

- non-linear (including especially quadratic Cohen's, affinity and hyperbolical transformations, or other special proceedings).

The main disadvantage of all linear and time-frequency transformations is the fact that the resulting determination within the time and frequency is limited by so called Heisenberg's principle of indefinitely. The frequency signal component can be presented only inside the rectangle $\Delta t \times \Delta f$ given in timefrequency area ( $\Delta t$ represents minimal time interval - time step, $\Delta f$ represents minimal frequency interval - frequency step). This fact may be limited in many applications. That is the reason why it is necessary to look for more precious proceedings in some cases. Especially, the non linear time-frequency transformation belongs to them.

Within non-linear procedures the most suitable are especially quadratic methods. From the point of view of mathematical features there is a very important group of quadratic transformations represented by so called Cohen's transformations. These include Margenau-Hill transformation too.

The mathematical definition of this time-frequency transformation is given by the following relation [2],

$\operatorname{MHT}(t, f)=\int_{-\infty}^{\infty} \frac{1}{2}\left[\begin{array}{l}x(t+\tau) \cdot x(t)^{*} \\ +x(t) \cdot x^{*}(t-\tau)\end{array}\right] \cdot e^{-j \cdot \omega \cdot \tau} \cdot d \tau$,

\footnotetext{
* Jaroslav Smutny, Daniela Sadlekova

Institute of Railway Structures and Constructions, Faculty of Civil Engineering, Brno University of Technology, Czech Republic

E-mail: smutny.j@fce.vutbr.cz
} 
where $t$ is time, $\tau$ is the time transition, $f$ is the frequency, $x(t)$ represents the time signal, symbol '*' represents the complex conjunction, $\mathrm{j}$ imaginary unit, $\omega=2 \cdot \pi \cdot f$ is angular frequency, and $\operatorname{MHT}(t, f)$ is the time frequency representation of the signal. Let us note that this refers to a complex transformation which, from the viewpoint of processing the measured signals has many suitable properties, offers an excellent resolution both in the time and in the frequency regions.

Generally, theory of non-linear time-frequency transformations is based on quantity mechanics and for derivation and calculation there can be used operator's theory. The theory assumes that every quadratic, time and frequency invariant transformations can be expressed by the relation

$$
\begin{aligned}
& C T_{x}(t, f)=\int_{-\infty}^{\infty} \int_{-\infty}^{\infty} A_{x}(\theta, \tau) \cdot \psi(\theta, \tau) . \\
& \cdot e^{-j \cdot \theta \cdot t} \cdot e^{-j \cdot \tau \cdot \omega} \cdot d \theta \cdot d \tau
\end{aligned}
$$

where $C T_{x}(t, f)$ is the time frequency representation of the signal, the symbol $\tau$ represents time-shifting, $\mathrm{j}$ imaginary unit, $\omega$ is angular frequency, $\theta$ is frequency shifting, $\psi(\theta, \tau)$ is the kernel function of particular time-frequency transformation and $A(\theta, \tau)$ represents time-frequency autocorrelation function. This function is given by the equation

$$
A_{x}(\theta, \tau)=\int_{-\infty}^{\infty} x\left(t+\frac{\tau}{2}\right) \cdot x^{*}\left(t-\frac{\tau}{2}\right) \cdot e^{j \cdot \theta \cdot t} \cdot d t .
$$

It is worth noting that this "narrow-band" function is complex and represents measure of time-frequency correlation of signal, or expresses the degree of similarity between the signal and its shifted version in time-frequency plane. In essence it represents the time-frequency autocorrelation function.

Kernel function $\psi(\theta, \tau)$ at the same time unambiguously determines the features of given transformation and in this way also determines the suitability of particular transformations for particular applications. It should be remarked that the kernel function of the Margenau-Hill transformation has got the form

$\psi(\theta, \tau)=\cos \left(\frac{\theta \cdot \tau}{2}\right)$

Multiplication of $A_{v}(\theta, \tau) . \psi(\theta, \tau)$ is also known under the name characteristic function. Because function $A_{x}(\theta, \tau)$ represents a bilinear operation on the processed signal and calculating the contributions arising from the above-mentioned cross-components which, in turn, deteriorate the resolution of the given transformation. This effect can be reduced by a suitable choice of kernel function. The kernel function then explicitly determines the properties of the transformation. In practice, the coefficients of the transformation from Cohen's class for discrete signals may be calculated by fast two-dimensional discrete Fourier transformation of the characteristic function $A_{x}(n, k) \cdot \psi(n, k)$.
To remove disturbing interference contributions (if their existence may significantly affect the analysis) their smoothed („pseudo“) version can also be defined by application of window function according to equation [1 and 3]

$\operatorname{PMHT}(t, f)=\int_{-\infty}^{\infty} \frac{h(\tau)}{2} \cdot\left[\begin{array}{l}x(t+\tau) \cdot x(t)^{*}+ \\ x(t) \cdot x^{*}(t-\tau)\end{array}\right] \cdot e^{-j . \omega . \tau} \cdot d \tau,(5)$

where function $h(\tau)$ represents suitable window function, $t$ time, $\tau$ the time transition, $\omega$ is angular frequency, $x(t)$ represents the time signal, symbol ' ' represents the complex conjunction, $\mathrm{j}$ is imaginary unit, and $\operatorname{PMHT}(t, f)$ is the time frequency representation of the signal.

The spectrograms are gained by the representation of the calculated values of Margenaau-Hill transformation or of the amplitude time-frequency spectrum in the graph. These spectrograms can also be represented in the three-dimensional space (frequency, time, amplitude or the spectral output density etc.). Alternatively, a two-dimensional representation by means of density spectrograms is also used in which an amplitude or a value of the spectral function of a certain shade is added to. When analysing some complex signals, it is appropriate to supplement the time frequency representation of spectrum with possible frequency and time sections. Then, these sections offer a distinctive graphic support in the analysis of the results of the time frequency analysis.

\section{Case study}

The next part of this paper describes the application of Margenau Hill transformation on the railway engineering issue. The maintenance and its cost minimization is one of the most discussed problems of the railway track nowadays. That is the reason why it is necessary to pay attention to diagnostics of the railway tracks and to assess steps which will lead to cost minimization. The effective diagnostic tools include analysis of the dynamic effects acting on the railway superstructure during train passing [4].

This is related mainly to the parts of aborted continuity and the parts where stiffness of the single-phase railway is changed as these are the parts where the advanced dynamic effects occur. As a result an uneven ballast settlement, gravel crushing and defects on the running surface occur. The common crossings are constructions with interrupted running surface; as a consequence they belong to the most stressed parts of the railway transport corridor from the point of view of dynamic effects. Their correct function and on-time maintenance have the principal impact on the safety of railway traffic. The most stressed point of crossing construction is the frog part consisting of wing rails and crossing nose followed by crossing rails (Fig. 1). 
The most important place of this part from the point of view of dynamic effects is the area of transmission from the wing rail to the crossing nose where the dynamic impact occurs. This impact is transmitted through the sleeper to the ballast which is thereby extremely stressed; which leads to grinding of ballast under the sleeper. As a consequence there is a degradation of the ballast shape and following weak support of the crossing. If the crossing is not supported enough, the geometry of the transmission from the wing rail to crossing nose collapses and the whole process of degradation continues very fast. That is the reason why it is necessary to pay extra attention to these parts of the railway track.

The measurement and the evaluation of the dynamic effects acting on the common crossings introduce the issue which has not been complexly solved in the Czech Republic yet. Therefore, the team of authors has compiled a comprehensive methodology of the measurement and evaluation of the dynamic effects acting on the crossings. This methodology (as Fig. 1 shows) consists of three parts. The measurement of moving behaviour is included in the first part. The measurement of moving behaviour represents the analysis of the shifts of particular parts of the crossing (vertical displacement of the sleeper under the crossing part and mainly the vertical shifts along the crossing part) under the load. For this the induction sensors are used. The second part of the methodology consists of measurement of vibrations spreading around the crossing part and especially the effects of vibrations on the ballast. The third part involves the measurement of the loading of rail. A part of the methodology is also the application of time-frequency analysis of the measured data.

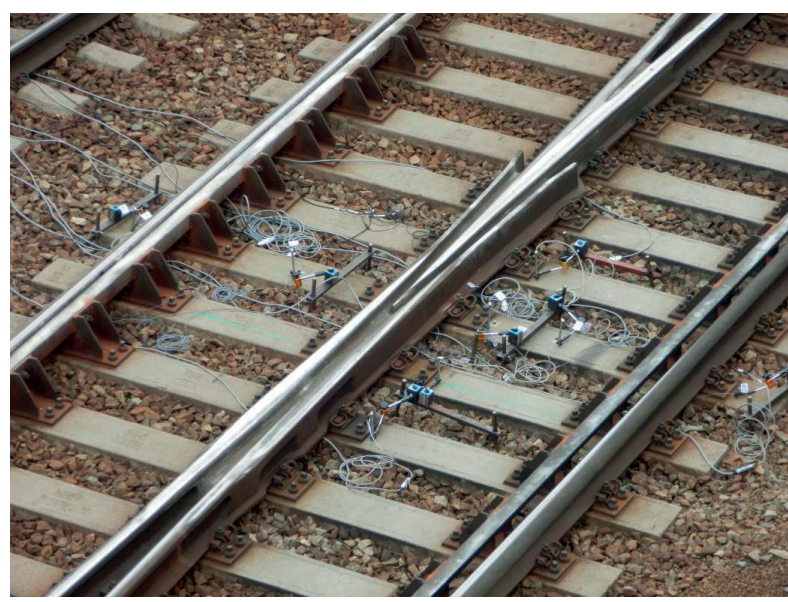

Fig. 1 General view of a frog including distributed sensors

The most important part of the methodology is devoted to the analysis of transmission of vibrations from the rail through the sleeper to the ballast caused by running wheelset of the railway vehicle. For measurement of parameters characterizing the dynamic effects the piezoelectric vibration acceleration sensors were used. The sensors are placed in such a manner that it's possible to follow up spread of vibration energy through the whole system. Three-axis accelerometer placed on flange of the wing rail detects the magnitude of dynamic impact which is acting on crossing part (Fig. 2, sensor A4ZA5XA6Y). Vertical dynamic impact posed on crossing part is detected in vertical direction, or the part which is transmitted to flange of the wing rail. According to the condition of transmission geometry there is also a part of dynamic impact carried out in longitudinal direction. In transversal direction the wheelset is led by the check rail, so the side impact is very small. Nevertheless, it is interesting to monitor this branch from the perspective of the fluency of unit train passage through the crossing, therefore, it is also a part of the suggested methodology.

The other sensors are one-axis sensors and are placed on such positions to best detect spreading of dynamic impact from the rail through the sleeper and into the ballast. Sensor A3Z is placed on the bearer nearest to the crossing nose and sensor A0Z is on the measure bar embedded to the ballast close to crossing nose.

The chosen crossings for measurement and analysis of dynamic effects were the fix common crossings of turnout number 59 (crossing inserted in 2009) and number 63 (crossing inserted in 2012) in station head of the railway station Chocen. The turnouts track systems are the same and both of them are a part of one common crossover. In addition to this, they are run by the same type of trains in trailing direction. These constructions were chosen with the aim to compare the dynamic effects in the crossing in various stages of wear.

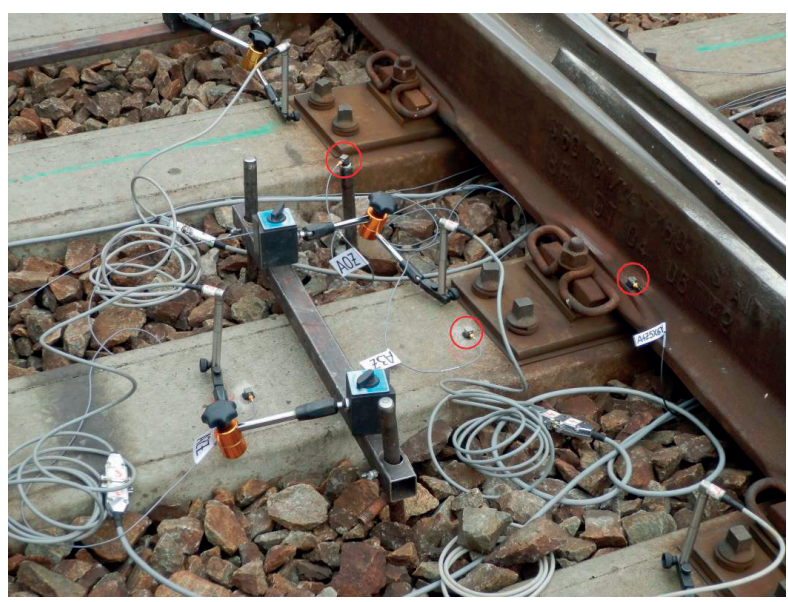

Fig. 2 Detail of a crossing part with vibration and shift sensors

For presentation of comparison of the dynamic loading on crossings the authors chose the unit train Pendolino with travel speed of $160 \mathrm{~km} / \mathrm{h}^{-1}$. Due to scope of this paper we will show only the evaluation from the sensor $\mathrm{A} 3 \mathrm{Z}$ installed on the bearer under the crossing nose.

For the illustration of the analysis, figures which consist of a triplet of graphs were used. The upper graph represents time course of vibration acceleration. The left graph shows the amplitude spectrum of the vibration response calculated by 
direct application of the Fourier transform on the measured signal. The middle graph is a $3 \mathrm{D}$ display of time-frequency amplitude spectrum of vibration response. The values of vibration acceleration in the decibel scale are shown in the middle graph as different colour areas while the maximum value is black.

Graphs in Fig. 3 represent analysis of passing of unit train Pendolino through turnout number 59 and graphs in Fig. 4 turnout number 63. Using the graphs of time courses (Figs. 3 and 4 , the upper graph) we are able to find out that the maximal and minimal values of vibration acceleration measured on turnout No.59 are higher than values on turnout No.63. These parameters provide information about unroundness of wheels, defective or overloaded axle or defect on the running surface of the rail. In our case they indicate a higher wear of the crossing nose of turnout 59 and thus also its worse transition geometry.

The left graph in Figs. 3 and 4 presents the amplitude spectrum of the vibration acceleration calculated from the measured signals by application of the Fourier transform. Amplitude spectra are presented in the frequency range of $1 \mathrm{~Hz}$ to $1000 \mathrm{~Hz}$. It is also important to say that for dynamic loading of structural components of the railway superstructure are significant frequencies up to $600 \mathrm{~Hz}$. From comparison of the both left graphs in Figs. 3 and 4 it is evident that vibration actions at the turnout No.59 occur in the wider frequency spectrum than at the turnout No.63. The crossing No.63 has its important components of the amplitude spectrum in frequency of up to $200 \mathrm{~Hz}$ while the crossing No.59 has significant frequency components in up to $600 \mathrm{~Hz}$, divided into more significant frequency clusters. This fact reflects the more complicated vibrational actions in the transition area of this crossing and also their higher energy. Within the analysis a decibel scale in the range $200-240 \mathrm{~dB}$ with a threshold value $1 \mathrm{e}^{-6} \mathrm{~m} / \mathrm{s}^{-2}$ was used.

To evaluate the vibration acceleration in the time-frequency area density spectrograms calculated by Margenau-Hill transformation were used (Figs. 3 and 4, the middle graph). Extreme values of vibration amplitude spectrum are shown in the graph in the form of red areas corresponding to the time intervals of wheel (wheelset) passing over the sensors. Both middle graphs in Figs. 3 and 4 show that the time occurrences of significant frequency components in the spectrum are different from each other. It is, therefore, well possible to identify at which moment there was an action (which wheel or axle caused it) and which frequency components correspond to this action. Time-frequency analysis therefore very well and accurately complements the conclusions derived from the time and frequency analysis.

\section{Conclusion}

The permanent pressure on increasing the transport speed and the operational loading of tracks causes a magnificent development of new technologies in the railway traffic. The development and the application of new experimental procedures for the evaluation of the quality and usefulness of particular structural solutions must be in accordance with this trend.

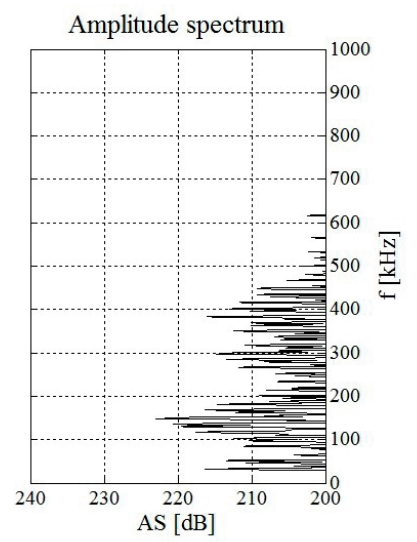

Pendolino set, turnout N. 59, bearer, vertical direction
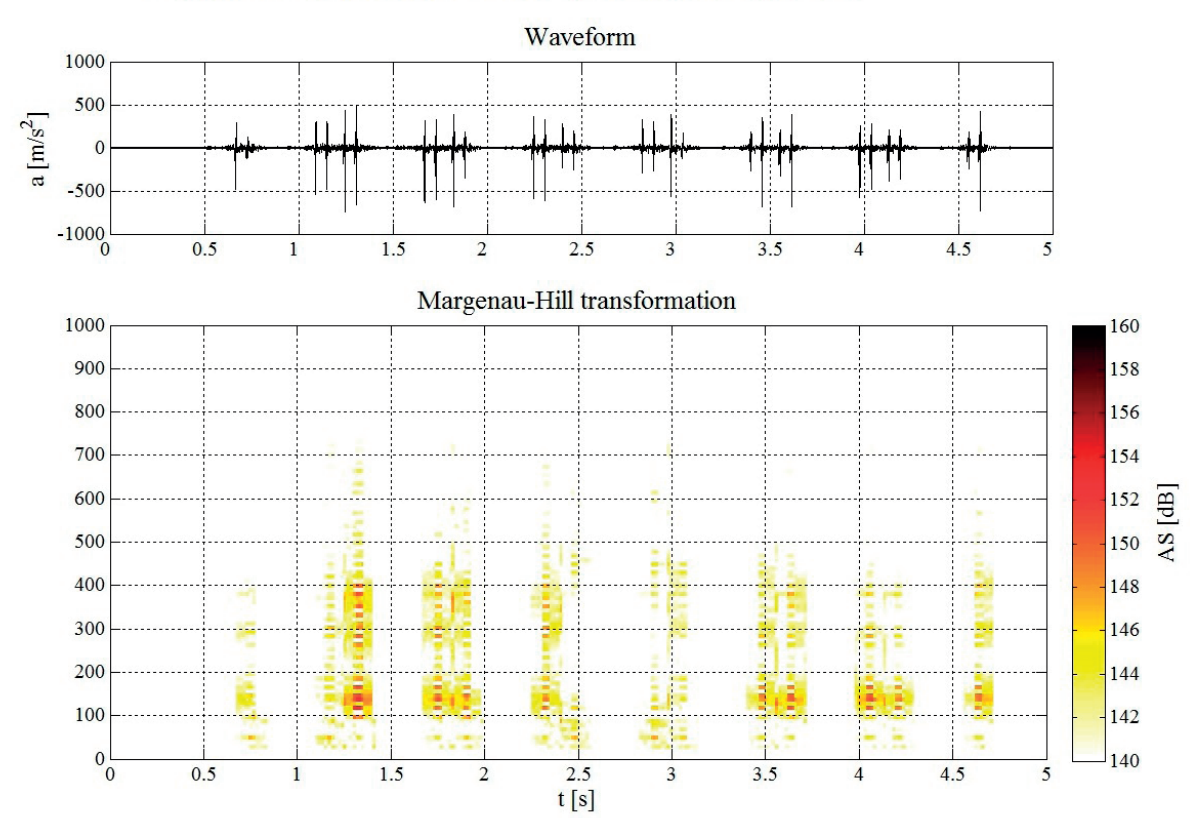

Fig. 3 Time history of acceleration, amplitude spectrum and jointed time-frequency spectrum in vertical direction for turnout No. 59 
Pendolino set, turnout N. 63, bearer, vertical direction
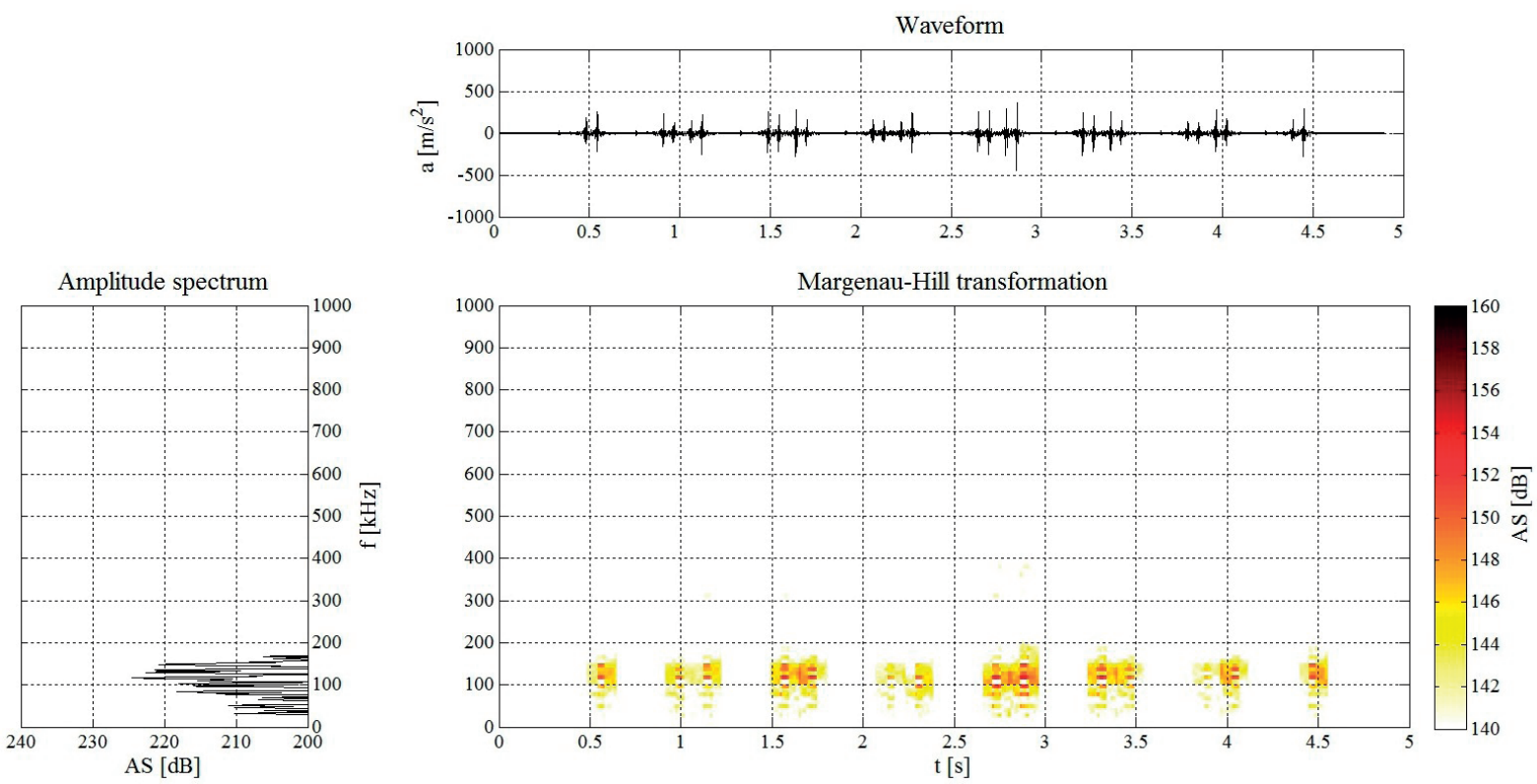

Fig. 4 Time history of acceleration, amplitude spectrum and jointed time-frequency spectrum in vertical direction for turnout No. 63

Especially methods of time-frequency analyses comparing to other methods distinguish information about the given technical activity so that they determine the time localization of frequency components, i.e. they determine the size of amplitude spectra on individual frequencies in respective time moment. It is, therefore, possible to state that the measurement and analysis of technical signals with the use of time-frequency methods provides mainly a new view to transition and non-stationary characteristics of measured structures.

Based upon the analyses made it is possible to say that the used methods offer good results and conclusions. The measured and calculated values prove to be sufficiently accurate and have testing capabilities. In conclusion, it should be stated that modern means of the time and frequency signal analyses (especially then Margenau-Hill transformation), perfectly contributed to the highquality processing of the data measured.
Generally, characteristic feature of presented non-linear transformations (including Margenau-Hill transformation) is the fact that their resulting resolution in time and frequency is not limited by Heisenberg principle of indefiniteness. This fact includes the high resolution ability in time-frequency level, which results in "precise" localization of important frequency components in time. The elaboration and generalization of the theory of non-linear time-frequency transformations contributed and will contribute to formation of further procedures with more advantageous properties.

\section{Acknowledgments}

This article was supported by the project FAST-S-14-2452, "The study of dynamic effect taking place in the switches by Margenau-Hill distribution”.

\section{References}

[1] POULARIKAS, A. D.: The Transform and Applications Handbook, IEEE Press, 1996.

[2] SMUTNY, J.: Measurement and Analysis of Dynamic and Acoustic Parameters of Rail Fastening, NDT \& E International - Independent Nondestructive Testing and Evaluation, ISSN 0963-8695, Elsevier, 2004.

[3] SMUTNY, J.; PAZDERA, L.: Jointed Time and Frequency Transforms in Testing Material Defects, Engineering Mechanics, ISSN 1802-1484, Engineering Academy : Brno, 2007.

[4] MORAVCIK, M.: Analysis of Vehicle Bogie Effects on Track Structure-nonstationary Analysis of Dynamic Response, Communications - Scientific Letters of the University of Zilina, vol. 13, No. 3, 2011, ISSN 1335-4205. 